\title{
Primary cutaneous leiomyosarcoma revealed by soft tissue tumor recurrence
}

\author{
Jean-Baptiste Andonaba1,4, Ollo Rolland Some ${ }^{2,4}$, Bakary Gustave Sanon ${ }^{3,4}$, \\ Boukary Diallo',4
}

${ }^{1}$ Department of Dermatology and Venereology, Sourô Sanou University Teaching Hospital of Bobo-Dioulasso (CHUSS), Burkina Faso, ${ }^{2}$ Department of Surgical Oncology, Sourô Sanou University Teaching Hospital of Bobo-Dioulasso (CHUSS), Burkina Faso, ${ }^{3}$ Department of General and Visceral Surgery, Sourô Sanou University Teaching Hospital of Bobo-Dioulasso (CHUSS), Burkina Faso, ${ }^{4}$ Polytechnic University of Bobo-Dioulasso (UPB), Higher Institute of Health Sciences (INSSA), Burkina Faso

Corresponding author: Prof. Jean-Baptiste Andonaba, E-mail: jb_andonaba@yahoo.fr

\begin{abstract}
Background: Primary cutaneous leiomyosarcoma (LCP) is a malignant tumor that can originate from smooth muscles (hair arrestor or vessels of hypodermic tissue). Recidivism rates vary by type. We report two particular cases by the volume of their recidivism and to underline the difficulties of management. Observations: ZA, 41 years old, was received after surgery for a voluminous, painless, firm, mobile and ovoid mass of $7 \mathrm{~cm}$ on the knee. The tumor occupied mainly the superficial and reticular dermis. It did not have immunohistochemistry but a re-reading of the blade concluded at diagnosis. The assessment of the extension did not objectify anything and the patient lost sight of. KM, 36 years was received 3 months after surgery, under the same conditions for a voluminous ulcerous burgeoning tumor, oblong $9 \mathrm{~cm}$ long axis of the shoulder curve. The histopathological lesions were extended to the subcutaneous tissue. The extension report showed lymphadenopathy, hepatic and pulmonary metastases. The patient underwent clean surgery associated with adjuvant chemotherapy. Conclusion: These relapses have no reliable information on the prognostic factors of their initial management. Nevertheless, the major factor of recurrence remains the tumor clearance, probably not obtained during the first excision. Close multidisciplinary collaboration is essential for the appropriate management of LCP.
\end{abstract}

Key words: Cutaneous leiomyosarcoma; Surgery; Recurrence

\footnotetext{
How to cite this article: Andonaba J-B, Some OR, Sanon BG, Diallo B. Primary cutaneous leiomyosarcoma revealed by soft tissue tumor recurrence. Our Dermatol Online. 2017;8(4):449-452.

Submission: $26.03 .2016 ; \quad$ Acceptance: 30.05 .2017

DOI: 10.7241 /ourd.20174.127
} 


\title{
Léiomyosarcomes cutanés primitifs révélés par une récidive tumorale des tissus mous
}

\section{Jean-Baptiste Andonaba ${ }^{1,4}$, Ollo Rolland Some ${ }^{2,4}$, Bakary Gustave Sanon ${ }^{3,4}$, Boukary Diallo ${ }^{1,4}$}

${ }^{1}$ Department of Dermatology and Venereology, Sourô Sanou University Teaching Hospital of Bobo-Dioulasso (CHUSS), Burkina Faso, ${ }^{2}$ Department of Surgical Oncology, Sourô Sanou University Teaching Hospital of Bobo-Dioulasso (CHUSS), Burkina Faso, ${ }^{3}$ Department of General and Visceral Surgery, Sourô Sanou University Teaching Hospital of Bobo-Dioulasso (CHUSS), Burkina Faso, ${ }^{4}$ Polytechnic University of Bobo-Dioulasso (UPB), Higher Institute of Health Sciences (INSSA), Burkina Faso

Corresponding author: Prof. Jean-Baptiste Andonaba, E-mail: jb_andonaba@yahoo.fr

\begin{abstract}
RESUMEN
Background: Le léiomyosarcome cutané primitif (LCP) est une tumeur maligne qui peut provenir des muscles lisses (arrecteur poil ou vaisseaux du tissu hypodermique). Les taux de récidive varie selon le type. Nous rapportons deux cas particuliers par le volume de leur récidive et pour souligner les difficultés de prise en charge. Observations: ZA, $4 l$ ans, a été reçue après chirurgie initiale pour une volumineuse masse indolore, ferme, mobile et ovoïde de $7 \mathrm{~cm}$ sur le genou. La tumeur occupait principalement le derme superficiel et réticulaire. Il n'a pas eu d'immunohistochimie mais une relecture de la lame a conclu au diagnostic. Le bilan d'extension n'a rien objectivé et la patiente perdue de vue. KM, 36ans a été reçu 3mois après une chirurgie, dans les mêmes conditions pour une volumineuse tumeur ulcérobourgeonnante, oblongue de $9 \mathrm{~cm}$ de grand axe du galbe de l'épaule. Les lésions histopathologiques s'étendaient jusqu'au tissu sous-cutané. Le bilan d'extension a montré des adénopathies et des métastases hépatiques et pulmonaires. Le patient a subi une chirurgie de propreté associée à une chimiothérapie adjuvante. Conclusion: Il s'agissait de récidives sans informations fiables sur les facteurs pronostiques de leur prise en charge initiale. Néanmoins le facteur majeur de récidive reste la clairance tumorale, probablement non obtenue lors des premières exérèses. Une étroite collaboration multidisciplinaire est indispensable pour la prise en charge adaptée des LCP.
\end{abstract}

Key words: Léiomyosarcome cutané; Chirurgie; Récidive

\section{INTRODUCION}

Le léiomyosarcome cutané primitif (LCP) est une tumeur maligne rare. Il représente 2 à 3\% de l'ensemble des sarcomes des tissus mous [1]. Ils peuvent provenir du muscle lisse arrecteur du poil à l'origine du léiomyosarcome dermique encore appelée léiomyosarcome cutané ou des muscles lisses des vaisseaux du tissu adipeux donnant le léiomyosarcome sous cutané ou hypodermique. Les taux de récidive, métastase et mortalité sont moindres pour le premier type selon plusieurs études rétrospectives et les revues de cas [2]. Si les données sont peu fréquentes dans la littérature du fait de la rareté de ce cancer cutané, elles sont inexistantes en Afrique subsaharienne. Nous rapportons deux cas particuliers par le volume de leur récidive pour rapporter les difficultés diagnostiques et les facteurs de récidive monstrueuse.

\section{CASE REPORT}

ZA, ménagère de 41 ans, 2 mois après chirurgie exérèse a été reçue dans notre service pour une volumineuse

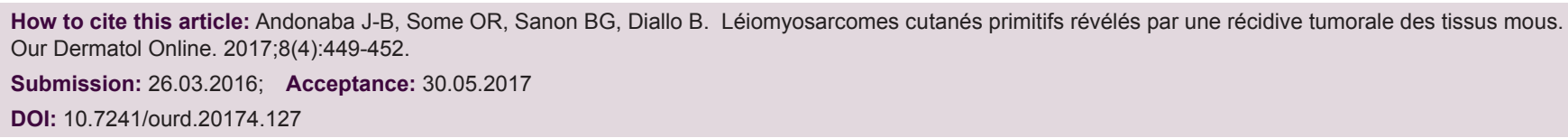


masse indolore ferme ovoïde de $7 \mathrm{~cm}$ de grand diamètre sur le genou mobile par rapport au plan profond (Fig. 1). L'histopathologie a montré des lésions avec un modèle de croissance nodulaire. La tumeur était confinée à la peau et occupait principalement le derme superficiel et réticulaire. Il n'a pas eu d'immunohistochimie mais une relecture de la lame a conclu au diagnostic. Le bilan d'extension ne retrouvait pas de métastases. La patiente a été perdue de vue.

KM, ouvrier de 36 ans a été reçu 3 mois après une chirurgie pour tumeur ulcérée dans les mêmes conditions que le premier cas pour une volumineuse tumeur oblongue de $9 \mathrm{~cm}$ de grand axe du galbe de l'épaule présentant une zone de $3 \mathrm{~cm} \mathrm{X} 4 \mathrm{~cm}$, ulcérobourgeonnante (Fig. 2). La masse est aussi mobile par rapport au plan profond. Les lésions histopathologiques avaient un aspect comparable à celles du premier cas avec un siège dépassant le derme superficiel et réticulaire pour atteindre le tissu sous-cutané. Le bilan d'extension comportant une radiographie thoracique et une échographie abdominopelvienne a montré des adénopathies et des métastases hépatiques et pulmonaires. La patiente a subi une chirurgie de propreté associée à une chimiothérapie adjuvante à base de la doxorubicine et sels de platines et confié à un réseau de soins palliatifs.

\section{DISCUSSION}

Peu de cas de LCP sont rapportés notamment les cas de récidive dont les facteurs sont intimement liés à la clairance tumorale lors de la chirurgie initiale. LCP est le troisième cancer après l'histiocytofibrome fibreux malin et le liposarcome [3]. La tumeur survient généralement entre 50 et 70 ans avec une prédominance masculine $[1,5]$. Nos cas avaient un âge plus jeune (36 ans et 4 lans), situation retrouvée dans les séries d'Auroy, Bernstein et al. [4,6]. Laspect clinique n'est pas spécifique avec une large gamme de diagnostics différentiels: le carcinome à cellules squameuses, le mélanome achromique, et le carcinome baso-cellulaire [7]. Le LCP est généralement considérée comme un nodule solitaire; le nodule peut être lobulé, pédiculée ou ombiliquée avec une surface qui peut être lisse, indurée, ulcérée, écailleuse, verruqueux ou hémorragique [8]. Nous avons observé le caractère solitaire, nodulaire à surface lisse et ulcérée. C'est une masse à croissance rapide et le pronostic est sombre si la masse $>5 \mathrm{~cm}$ [8]. Pour nos deux cas les tailles sont volumineuses peu rencontrés dans la littérature

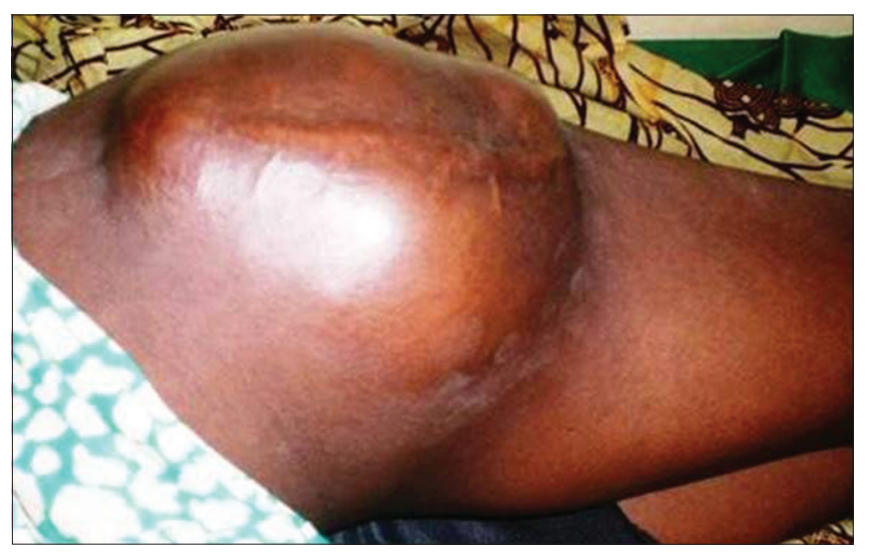

Figure 1: Récidive d'un léiomyosarcome du genou.

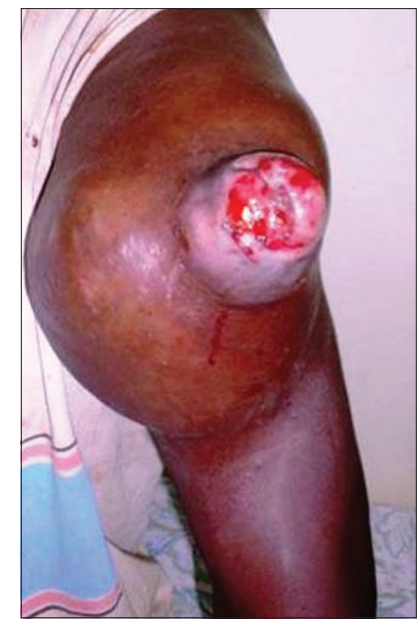

Figure 2: Récidive d'un léiomyosarcome de l'épaule: tumeur ulcérobourgeonnante.

même en cas de récidive. De plus une pseudocapsule inflammatoire fibreuse constitue une fausse limite qui trompe les opérateurs les moins avisés [9]. Ces cas illustrent la difficulté de définir avec précision les limites de la tumeur sur la base de l'imagerie seule et la clinique. Nos deux observations constituent des cas de récidive sans qu'on puisse avoir des données fiables sur les facteurs pronostiques de leur prise en charge initiale. Néanmoins le facteur majeur de récidive reste la clairance tumorale qui n'a pas pu être probablement obtenu lors de premières exérèses. Les conditions de leurs réalisations à savoir dans les structures sanitaires périphériques par des agents de santé moins qualifiés en chirurgie cancérologique expliquent leur exérèse par énucléation. Alors que l'on sait que le risque de réchute n'est pas nul même en cas de resection $\mathrm{R} 0$ des LMS dermiques qui récidivent peu [10]. C'est peut être ce qui explique que certains auteurs militent pour un une exérèse large avec $3-5 \mathrm{~cm}$ de clairance tumorale et une profondeur qui comprend le tissu sous-cutané et le fascia [11]. Cependant devant la localisation au 
niveau des extrémités et dans le souci de préserver le maximum de tissu sain certaines études rétrospectives récentes $[2,10]$ et une métaanalyse [12] prône une marge d'au moins $1 \mathrm{~cm}$ pour réduire considérablement le risque de récidive locale et à distance. Lexcision locale sans marges adéquates conduit à la récidive et augmente le risque de maladie métastatique et de mortalité. Nous notons un cas de métastase ganglionnaire et hépatique chez le cas de leiomyosarcome sous cutané. En effet ce risque semble plus élevé indépendamment de la marge de résection, sur les tumeurs sous cutanées [2]. La chirurgie avec un examen extemporané histologique des limites permet d'éviter des marges de résection proches de la tumeur [9]. Cependant l'extemporané n'est pas une recommandation car peu fiable dans les sarcomes. Un autre procédé de traitement est une opération micrographique de Mohs pour assurer l'élimination complète de la tumeur [13] qui serait plus indiqué sur des tumeurs initiales de petites tailles et/ou de localisation préjudiciable pour des exérèses larges. Il nécessite une pratique courante pour la lecture microscopique. Pour nos cas de récidive la taille et l'existence de métastases dans un cas et l'inexpérience de la pratique constituent individuellement des contre -indications. La Cryochirurgie a également été utilisée chez des patients âgés. Les thérapies adjuvantes comprennent la radiothérapie, la chimiothérapie et la thérapie de cobalt supervoltage [8]. Cependant le LCP a été rapporté comme radiorésistante; également la chimiothérapie avec la doxorubicine a échoué dans certains cas [1]. Si les léiomyosarcomes cutanées sont reconnus avec un taux de récidive locale de 30-50\% et rarement métastaser, les léiomyosarcomes souscutanées se reproduisent dans un maximum de $70 \%$ et le taux métastatique a été rapportée chez 30-40\% des cas selon certains auteurs [14].

\section{CONCLUSION}

Les récidives locales et les métastases à distance peuvent survenir des mois ou années après l'excision initiale. La corrélation anatomoclinique et immunohistochimie sont obligatoires pour le diagnostic définitif.
Limportance des besoins en suivi à long terme doit être soulignée pour les LCP. Une étroite collaboration entre dermatologues, chirurgiens, cancérologues et pathologistes est indispensable pour la prise en charge adaptée de cette affection au CHU Sourô Sanou de Bobo-Dioulasso.

\section{REFERENCES}

1. Lin JY, Tsai RY. Léiomyosarcome sous-cutanée sur le visage. Dermartol Surg. 1999;25:489-91.

2. Winchester DS, Hocker TL, Brewer JD, Baum CL, Hochwalt PC, Arpey CJ, et al. Leiomyosarcoma of the skin: Clinical, histopathologic. J Am Acad Dermatol. 2014;71:919-25.

3. Hashimoto H. Leiomyosarcoma. Cancer. 1986;57:2077-88.

4. Auroy S, Contesso G, Spatz A, Genin J, Margulis A, Lecesne A, et al. Léiomyosarcomes Primitifs: 32 CAS. Ann Dermatol Venerol. 1999;126:235-42.

5. Stout A, Hill W. Leiomyosarcoma of the superficial soft tissues. Cancer. 1958;11:845-54.

6. Bernstein SC, Roenigk RK. Leiomyosarcoma of the skin: treatment of 34 cases. Dermatol Surg. 1996;22:631-5.

7. Kuflik JH, Schwartz RA, Rothenberg. Léiomyosarcome. J Am Acad Dermatol. 2003;48:51-3.

8. Vraa S, Keller J, Nielsen OS, Jurik AG, Jensen OM. Soft-tissue sarcoma of the thigh: surgical margin influences local crecurrence but not survival in 152 patients Acta Orthop Scand. 2001;72:72-7.

9. Dfouni N, Switzerland A. Léiomyosarcome. Radiology teaching files case, University Hospitals of Geneva. 2011. https://www.mypacs. net/cases/55465555.html.

10. Cochereau D, Battistella M, Morelot Q, Pagès C, Basset-Seguin N, Marco O, et al. Pronostic et risque de rechute locale dans les léiomyosarcomes cutanés sus-aponévrotiques en résection complète. Ann Dermatol Vénéréol. 2015;142:S453.

11. Poisson FS. sarcomes des tissus mous en dermatologie. Dermatol Surg. 1996;22:268-73

12. Jose Aneiros-Fernandez, Juan Antonio Retamero, Husein Husein-El Ahmed, Francisco Ovalle, Jose Aneiros-Cachaza. Primarycutaneous and subcutaneous leiomyosarcomas: evolutionand prognostic factors. Eur J Dermatol. 2016;26:9-12.

13. Huether MJ, JA Zitelli, Brodland DG. la chirurgie micrographique de Mohs pour le traitement des tumeurs à cellules fusiformes de la peau. J Am Acad Dermatol. 2001;44:656-59.

14. Pashaei S, Lind AC, Thomas AW, Faulkner-Jones BE. Léiomyosarcome récurrent de la peau. Pathol Case Rev. 2005;10:281-6.

Copyright by Jean-Baptiste Andonaba, et al. This is an open-access article distributed under the terms of the Creative Commons Attribution License, which permits unrestricted use, distribution, and reproduction in any medium, provided the original author and source are credited.

Source of Support: Nil, Conflict of Interest: None declared. 